\title{
COVID-19 and the Radiology Department: What We Know So Far
}

\author{
Sanya Vermani ${ }^{1} \cdot$ Aditya Kaushal $^{2} \cdot$ Jessica Kaushal ${ }^{3}$ \\ Accepted: 24 September 2020 / Published online: 2 October 2020 \\ (C) Springer Nature Switzerland AG 2020
}

\begin{abstract}
COVID-19 is a global healthcare pandemic that is now growing through nations across the world. The role of radiology is crucial, and a variety of guidelines have been published regarding the role of imaging. These aim to protect healthcare workers (HCWs) and the general public from exposure, while preserving critical radiology operations and conserving personal protective equipment (PPE) and other critical care resources during the COVID-19 pandemic. Fleischner Society published guidelines on indications of imaging various settings. These guidelines take into account resource availability, pre-test probability, degree of symptoms and risk factors, which is crucial for decision-making regarding need and indications of imaging. Mitigating steps and alternative approaches should be considered to provide the best care for patients while protecting all HCWs. Owing to overlap of COVID-19 imaging findings with other pathologies, standardized reporting acquires importance for risk assessment and effective communication of suspicious findings. RSNA followed by Dutch Radiological Society (NVvR) have published guidelines on standardized CT reporting for COVID-19, which show excellent inter-observer variability. Standardized reporting can provide guidance and confidence to radiologists as well as increased clarity to physicians through reduced reporting variability. The article discusses the published recommendations and aims to make radiologists aware of the protocols and guidelines that need to be followed in this ongoing public health crisis for effective patient care while protecting HCWs and conserving resources.
\end{abstract}

Keywords COVID-19 $\cdot$ Radiology $\cdot$ Imaging $\cdot$ Pandemics $\cdot$ Coronavirus

\section{Introduction}

In and around the Huanan seafood market in Wuhan, located in Hubei province in China, a cluster of cases with unexplained low respiratory infections with few developing acute respiratory distress syndrome and respiratory failure were detected in December 2019 [1,2]. On 31 December 2019, these were reported to the WHO Office in China. Published literature can trace the onset of symptomatic people back to early December 2019. Such first cases $(n=29)$ were labelled as "pneumonia of unknown aetiology" since they were unable to classify the causative agent. An ambitious outbreak investigation system was

Aditya Kaushal

drkaushalortho@gmail.com

1 All India Institute of Medical Sciences, Virbhadra Road, Rishikesh, India

2 Post Graduate Institute of Medical Education and Research, Sector-12, Chandigarh, India

3 Government Medical College, Circular Road, Amritsar, India coordinated by the Chinese Center for Disease Control and Prevention (CDC). The aetiology of this disease has been traced to a new coronavirus $(\mathrm{CoV})$ virus. The pathogen was identified on January 7, 2020, as a novel form of virus from the coronavirus family and was temporarily named by the World Health Organization (WHO) as '2019-nCoV.' On 11 February, it was formally called COVID-19 by the WHO [3]. It was formally declared a pandemic by the WHO on 11 March 2020. RT-PCR tests have shown high sensitivity and near-perfect specificity in a laboratory setting; however, a number of factors may adversely affect test sensitivity in clinical practice, including specimen adequacy, size, handling and specimen acquisition time $[4,5]$. False-negative RTPCR tests were reported in COVID-19 CT patients, who eventually tested positively with serial sampling [6]. Hence, imaging may acquire an important role in early cases or in resourceconstrained setting with paucity of available tests. Various guidelines have been published, which may aid in decision-making for the radiology department in regard to indications of imaging, stratification of risk for COVID19 on basis of computed tomography (CT) imaging findings in a standardized reporting system. This article will 
review the published guidelines in a serial manner, from handling of elective and emergency cases, precautions to be followed while imaging suspected or confirmed COVID 19 cases, indications for imaging in suspected/confirmed cases as well as imaging features of COVID-19 and risk stratification on CT.

\section{Precautions for Radiology Elective Imaging}

According to RSNA COVID-19 Task Force [7], all screening investigations (including coronary artery calcium scoring, mammography, DEXA scans and CT Colonography) may be postponed. RSNA COVID-19 Task Force recommends a triage of investigations to be carried out in three groups, based on agreement between the radiologist and the physician in charge.

- Category 1 (Elective/Non-Urgent): These do not impact patient outcome if postponed by up to $2-6$ months. These investigations can be delayed.

- Category 2 (Time Sensitive): It is possible to consider a short delay (up to 2-4 weeks) for these.

- Category 3 (Critical): These must be done promptly.

For the expected elective investigations, the patients must be checked for COVID-19 symptoms including fever, new cough, dyspnoea and/or breathlessness at the radiology front desk/entrance. Visitors who are accompanying patients must be forbidden, except in cases where they are critical to the ambulation of patients. Those found positive at screening must be masked in the waiting area and isolated from other patients. Portable imaging for these patients should be promoted where feasible. If the imaging is to be carried out in the main room, sufficient measures must be taken. Dedicated scanners may be assigned to positive or suspected patients with COVID-19 if resources allow [7].

\section{Precautions for COVID-19-Suspected/-Confirmed Cases}

Appropriate measures must be taken when a patient either shows the signs at screening or is a confirmed case outlined as below, with the personnel present in the room reduced to only those necessary for the procedure to be carried out. N-95 masks must be fit-tested to the workers. A significant problem in the radiology department occurs as few N95 masks have a metal seal which can result in seal loss in the MRI space. For these cases, non-ferrous N95 masks can be used.

Most cases require droplet/contact precautions that include

- Surgical masks to be worn by the patients.
- Staff to wear properly fitted N95 masks or surgical masks with face shields and contact protection gear (which includes gown and gloves).

For aerosol-generating procedures [8] (which include gastrointestinal fluoroscopic studies intubation/extubation, tracheostomy, lung biopsy, chest tube insertion, nasogastric tube placement, gastrointestinal stomies or stent placement), airborne precautions must be instituted, including

- Surgical masks to be worn by the patients.

- Staff to wear properly fitted N95 masks with face shields and contact protection gear (which includes gown and gloves).

Hand hygiene, which involves hand washing for $20 \mathrm{~s}$ with soap and water or using a hand sanitizer (with more than $60 \%$ ethanol or $70 \%$ isopropyl alcohol) should be practiced in all situations [9].

\section{Post-Imaging Cleaning}

Duration of the closure of the room after cleaning depends on exchange rates for the room air, having an inverse relationship [10]. Simple cleaning of equipment using quaternary wipes impregnated with ammonium/alcohol should be undertaken in every situation. There is no need for room closure if the air circulation is sufficient for droplet precautions. For airborne precautions, rooms with over 6 air exchanges an hour, which includes cleaning time, must be closed for $1 \mathrm{~h}$. Highefficiency particulate air filter (HEPA) can be used to improve air circulation and, where appropriate, to shorten the closure of rooms. This is however not compatible with MRI.

\section{Role of Imaging in COVID-19}

Portable chest radiography is the first-line imaging modality due to ease of accessibility and cleaning/disinfection. Although sensitivity is low in early cases and those with mild infection, it provides a quick diagnostic tool. Computed tomography (CT) is a highly sensitive modality, with a disadvantage that it requires disinfection measures after scan of every COVID-19-suspected/-positive patients, which can be time-consuming and troublesome especially in hospitals with a high case-load. This issue can be resolved by providing a dedicated CT scanner for COVID imaging, when feasible. In hospitals with only a single CT scanner, consecutive imaging of all COVID-19-suspected/-confirmed cases can be done followed by disinfection [11].

Fleischner Society guidelines [12] guide need and protocol of imaging in patients with varying grades of risk for COVID- 
19 with consideration to other factors including resource constraints, pre-test probability, disease severity and progression. Three clinical scenarios were outlined. First scenario includes patients with mild features of COVID-19 with any pre-test probability and no significant resource constraints. In such cases, imaging may be indicated in those test-positive/high pre-test probability patients with risk factors for disease progression. Imaging is also indicated in cases with worsening respiratory status, irrespective of risk factors, test-positive or pre-test probability.

Second scenario includes patients with moderate to severe features of COVID-19 with any pre-test probability and no significant resource constraints. In this scenario, imaging is recommended in all test-positive patients with further imaging recommended in case of worsening respiratory status. In testnegative patients, imaging is recommended as per standard clinical practice/existing guidelines to rule out alternate diagnosis if suggested.

Third scenario includes patients with moderate to severe features of COVID-19 with high pre-test probability and resource constraints. In this scenario, lack of resources may necessitate patient triage. Imaging is done in test-positive patients with worsening respiratory status. Based on availability of resources and clinical judgement, it may also be done in test-positive patients. In test-negative patients, existing guidelines should be followed to make imaging decisions to rule out alternate diagnosis.

Mild severity encompasses those with no obvious significant pulmonary dysfunction, as determined by the lack of hypoxaemia or significant dyspnoea. Moderate to severe disease severity is defined when hypoxaemia or moderate to severe dyspnoea is seen.

Background prevalence of the disease determines the pretest probability. It is low in sporadic transmission, medium in clustered transmission and high in community transmission [13].

Risk factors for disease progression include age $>65$ years, cardiovascular disease, diabetes mellitus, hypertension, chronic lung disease and immunocompromised status [14].

Hypoxaemia is the objective measure of worsening respiratory status, which necessitates imaging in various scenarios as described above.

Additional recommendations:

- Chest radiographs done on a daily basis are not indicated in stable intubated RT-PCR-positive patients as many studies show no benefit in outcome with daily versus ondemand or requirement-based imaging [15-18]. Further, daily imaging may increase exposure of HCWs and also consume personal protective equipment (PPE).

- CT imaging is indicated in recovered COVID-19 patient having persisting hypoxaemia as COVID-19 is a relatively new infection and imaging of recovered patients will aid in identifying the long-term sequelae/morphological alterations.

- COVID-19 testing is indicated in a patient with incidental findings of the disease on CT. Asymptomatic carriers, which contribute to $17.9-33.3 \%$ of all infections [19, 20], may show positive CT findings incidentally when being imaged for other causes. Although imaging findings of COVID-19 are relatively non-specific, they can be highly suggestive in a setting of high community transmission. As such asymptomatic patients may contribute to rapid spread, confirmatory testing by RT-PCR is essential in identification and prospective curtailment of disease spread.

To summarize the guidelines of Fleischner Society, imaging is not indicated as a screening test in asymptomatic individuals or those with mild symptoms unless risk for progression of disease is present. Indications of imaging include patients with moderate/severe symptoms irrespective of RTPCR test results, evidence of worsening respiratory status as assessed by hypoxaemia. It is also suggested to prefer chest radiography over CT in a resource-limited setting where access to CT may be variably limited.

\section{CT Imaging Findings and Standardized Reporting}

Characteristic imaging features of COVID-19 on CT include ground-glass opacities (GGOs) in a bilateral multi-lobar distribution with preferential posterior and peripheral involvement [21]. Patterns of GGOs typically seen include crazypaving, rounded or a linear morphology. Crazy paving pattern is a combination of GGOs with interstitial thickening [21,22]. Although it is a non-specific finding seen in many other pathologies, it is seen in COVID-19 predominantly in a peripheral distribution [22]. Another imaging feature is presence of a prominent/enlarged sub-segmental vessel (diameter $>3 \mathrm{~mm}$ ), which could be attributed to hyperaemia caused by COVID19 [21]. This differs from pulmonary involvement in other known coronavirus diseases, where constriction of pulmonary vasculature is seen [22]. Reverse Halo or Atoll sign, which refers to central area of ground-glass opacity surrounded by consolidation, has been reported in late stages of COVID-19 infection [22]. Consolidations with air bronchogram could be seen in those with rapid progression of pulmonary involvement, indicating alveolar filling with exudates with patent bronchioles. Interstitial septal thickening is commonly seen, with or without associated GGOs [22]. Bronchiectasis (especially tractional) and mediastina/hilar lymphadenopathy may be seen in advanced cases [22]. Cavitation in areas of GGOs and pleural and pericardial effusion could also be infrequently seen in advanced/critical cases [22]. 
A standardized reporting system for CT assessment of COVID-19 cases was developed by the Dutch Radiological Society (NVvR) [23] for structured reporting and communication. It divides the cases into 5 categories, ranging from CORADS 0 to CO-RADS 6. RSNA also published the RSNA Consensus on CT reporting [24] to facilitate standardized reporting and ease of communication of findings to the referring physician.

CO-RADS 0 is assigned when the scans are incomplete or have suboptimal quality such as those with respiratory motion artefacts.

CO-RADS 1, which indicates very low suspicion of COVID-19, encompasses findings including a normal scan or one which shows overt or clear findings of non-infectious pathology, which covers various other pathologies ranging from emphysema, lung tumours, fibrosis or perifissural nodules (Fig. 1). This coincides with the Negative for Pneumonia category of RSNA Consensus on CT reporting [24], which includes cases where no CT features to suggest pneumonia were seen.

CO-RADS 2, which indicates a low suspicion of COVID19 , encompasses imaging findings that are typical for infective causes, however not compatible with COVID-19. These

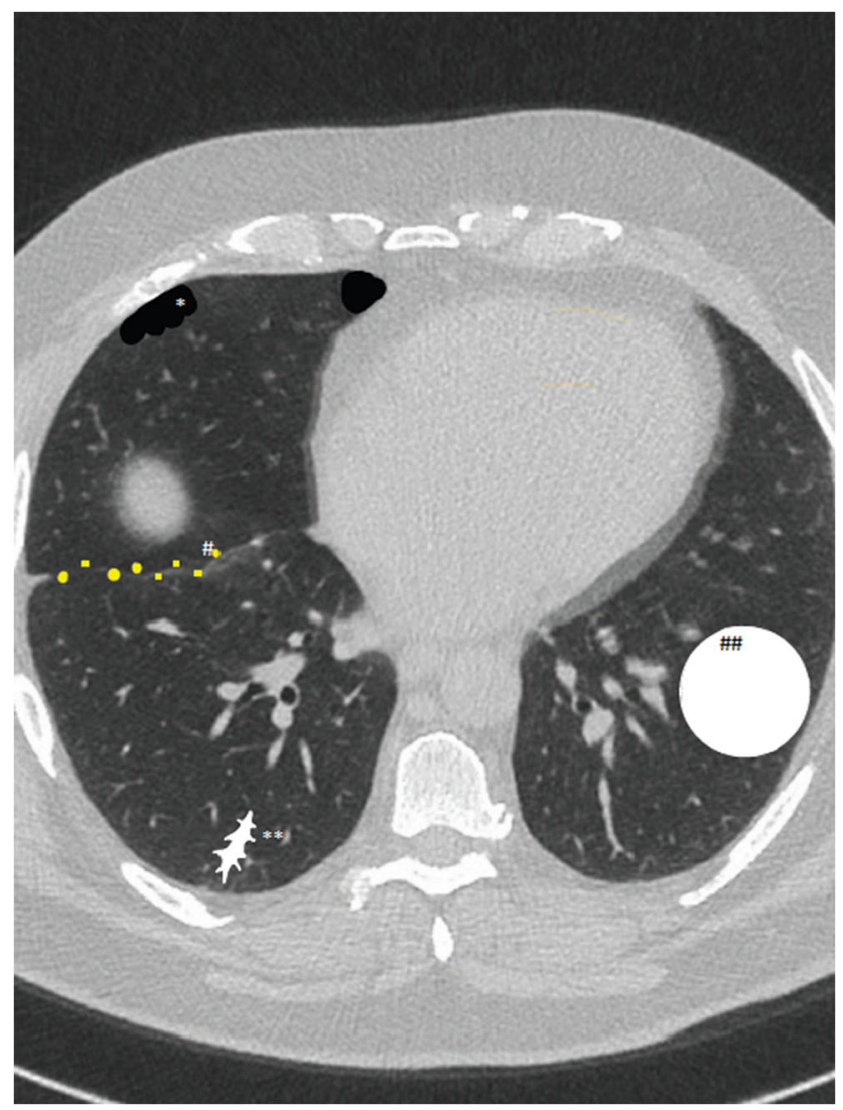

Fig. 1 Illustrated image depicting CT findings of CO-RADS 1. The single asterisk $(*)$ indicates emphysema; the single number sign (\#) represents perifissural nodules; the double asterisk $(* *)$ represents fibrosis; the double number sign (\#\#) indicates lung carcinoma may include bronchitis, infectious bronchiolitis as seen on imaging as centrilobular or tree-in-bud nodules, bronchopneumonia or lobar pneumonia, which may be seen on imaging as areas of segmental or lobar consolidation, and pulmonary abscess, which may be seen as lung cavitation (Fig. 2). Cases with smooth interlobular septal thickening may come under CO-RADS 1 if seen in conjunction with pleural effusion and suspicious for pulmonary oedema as CO-RADS is based on the pulmonary features of COVID-19 and not the cardiac features. However, in case ground-glass opacities that are suspicious for typical pulmonary involvement of COVID-19 are also present in conjunction with smooth interstitial thickening, then a CO-RADS 3 grade is advised. CO-RADS 2 coincides with Atypical Appearance of RSNA Consensus on CT reporting [24], which encompasses findings that may include areas of isolated consolidation in a lobar or segmental distribution, absence of ground-glass opacities, presence of small nodules in centrilobular distribution or lung cavitation.

CO-RADS 3 or equivocal scan includes findings like perihilar or homogeneous extensive ground-glass opacities. Sparing of few secondary pulmonary lobules may or may not be present. Ground glass in conjunction with smooth interstitial thickening with presence or absence of pleural

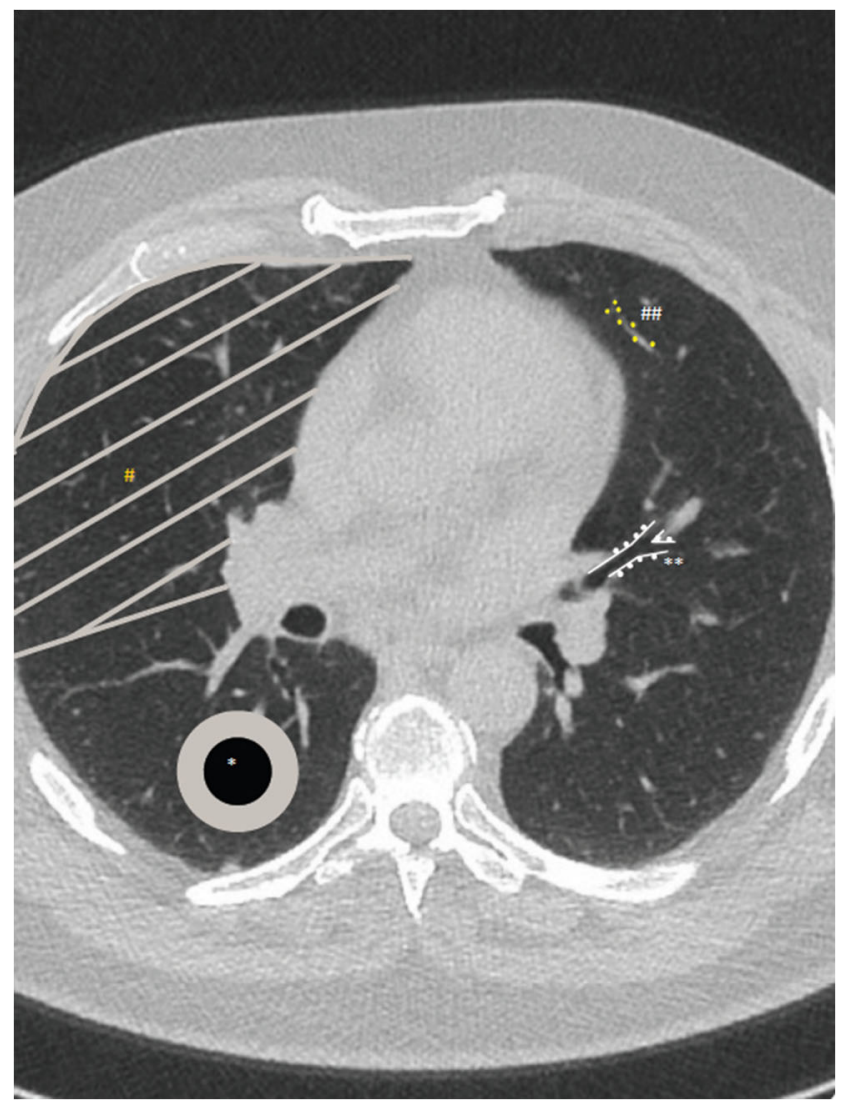

Fig. 2 Illustrated image depicting CT findings of CO-RADS 2. The single asterisk $\left(^{*}\right)$ indicates lung abscess; the single number sign (\#) indicates lobar consolidation; the double asterisk (**) indicates bronchitis; the double number sign (\#\#) indicates centrilobular nodules 
effusion without other classic CT findings of COVID-19 is also included in the CO-RADS 3 category (Fig. 3). This category also includes variable grades of ground-glass opacities that do not suit in the CO-RADS 2 or 4 categories, for example small ground-glass opacities, which are not seen in a centrilobular location (CO-RADS 2) or not situated close to the visceral pleural surfaces (CO-RADS 4). Additionally, it also includes patterns of consolidation seen in organizing pneumonia without other classical imaging features of COVID-19.

CO-RADS 4, which indicates a high suspicion of COVID19 , includes CT findings which may be typically seen in COVID-19, however may overlap with other causes of viral pneumonia. Findings are similar to CO-RADS 5 but are either not seen in contact with or near the visceral pleural surfaces or may be seen completely in one lung; these may be located a peribronchovascular pattern or may be seen in conjunction with other diffuse pre-existing lung pathologies (Fig. 4).

The category CO-RADS 5, which indicates a very high suspicion of COVID-19, includes the typical or classic findings. These include ground-glass opacities with presence or absence of accompanying areas of consolidation. These are situated in regions close to visceral pleural surfaces or the periphery including the lung fissures and show a multifocal

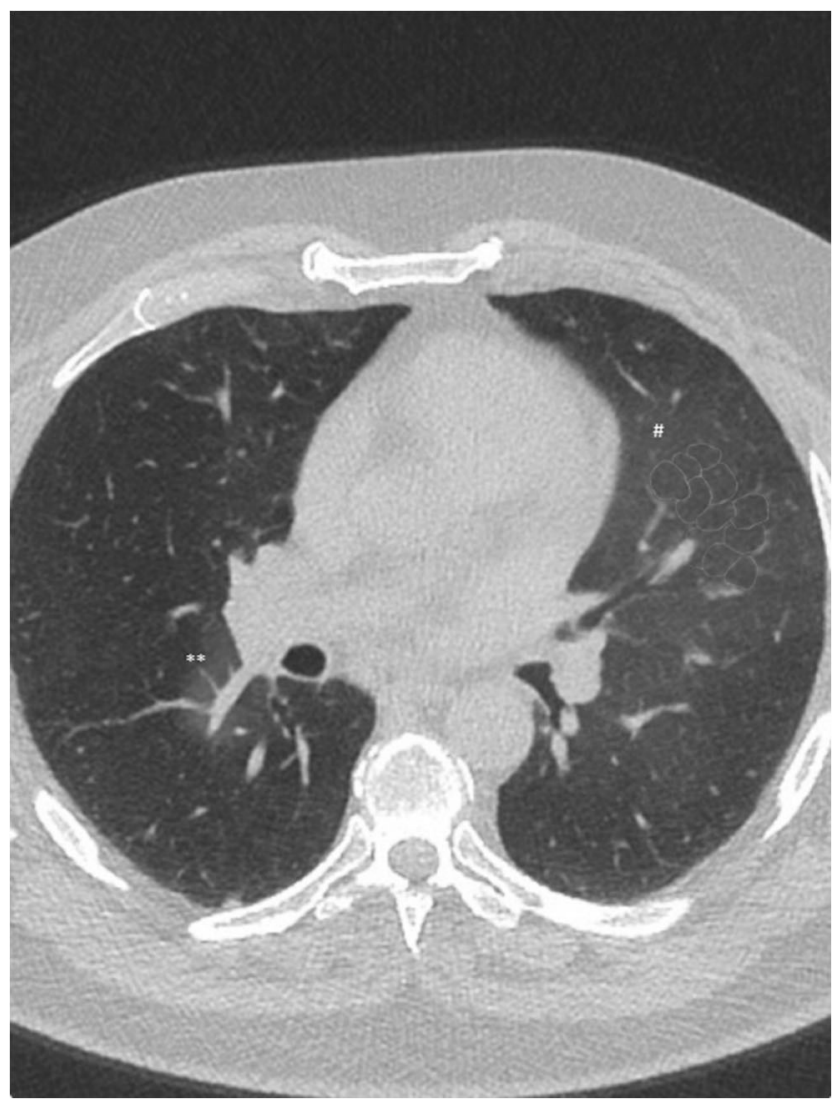

Fig. 3 Illustrated image depicting CT findings of CO-RADS 3. Groundglass opacities in a homogeneous extensive (number sign) or perihilar (double asterisk) distribution with smooth interstitial thickening

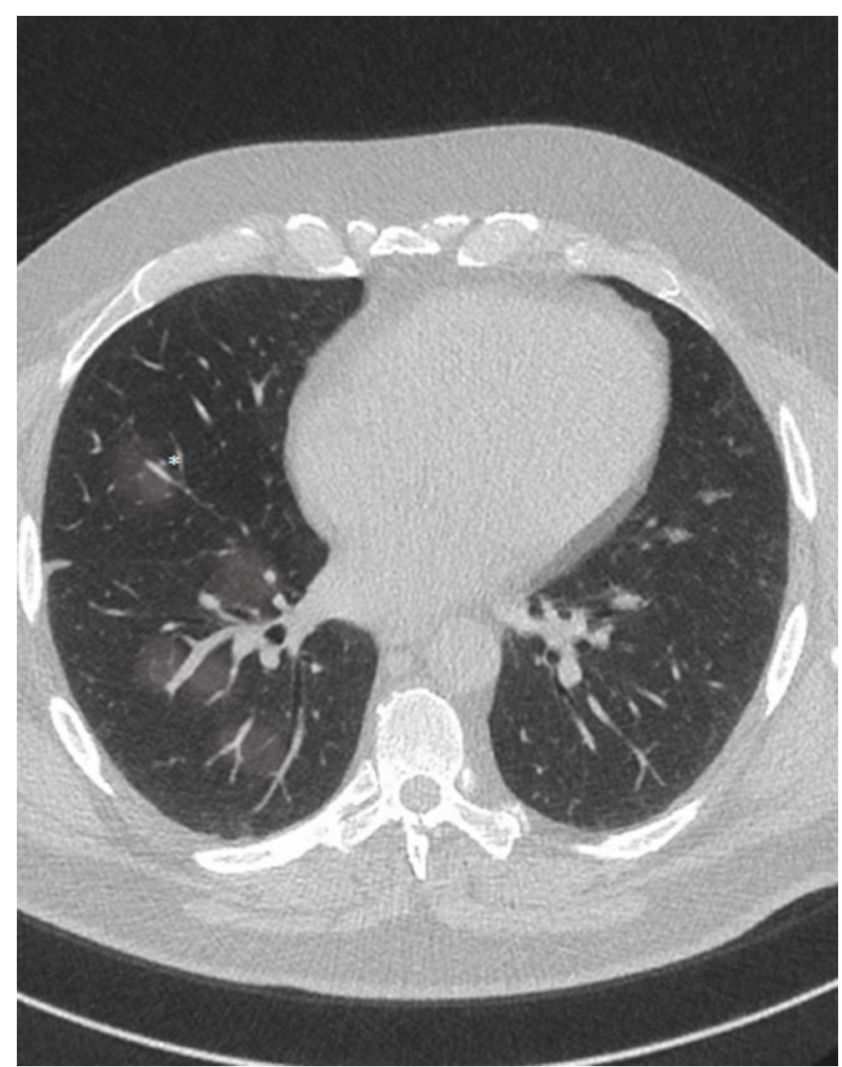

Fig. 4 Illustrated image depicting CT findings of CO-RADS 4. Groundglass opacities in a unilateral peribronchovascular (asterisk) distribution

bilateral pattern of distribution. Rather than only a peripheral location as was previously described, vicinity to the minor or major fissure is also typical. Areas of subpleural sparing can be seen (Fig. 5). Previously described predominance in lower lobes was removed as a necessary feature in CO-RADS. CORADS 4 coincides with the Indeterminate Appearance of RSNA Consensus on CT reporting [24], which includes ground-glass opacities with/without consolidations, lacking a specific distribution or occurring in a non-peripheral distribution and with non-typical shape or appearance.

The category CO-RADS 5 requires at least one of the following confirmatory patterns, which are seen as the disease progresses temporally. In the early stage, numerous areas of ground-glass opacities are seen, typically showing half round and unsharp or hazy margins; however, these may be accompanied by sharply marginated areas of ground-glass opacities which follow the outline of adjacent secondary pulmonary lobules. In the following stage, a crazy paving pattern may be seen, which shows interstitial (intralobular) septal thickening in conjunction with areas of ground-glass opacities. With further progression, progressive areas of consolidations may appear in the areas of ground-glass opacities. With further temporal progression are seen findings of organizing pneumonia having classic features including reverse halo or Atoll sign (consolidation surrounding areas of ground-glass opacities) or 


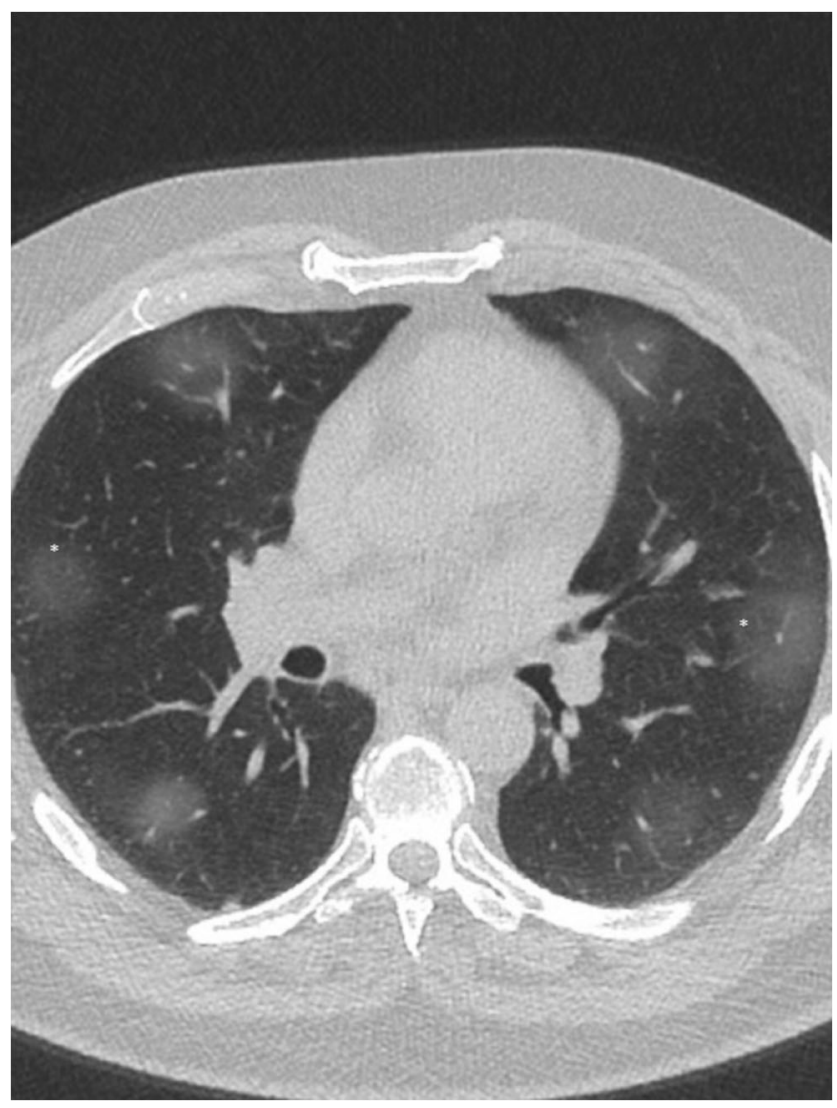

Fig. 5 Illustrated image depicting CT findings of CO-RADS 5. Groundglass opacities in a multifocal bilateral (asterisk) distribution

ground-glass opacities with extensive consolidations in subpleural regions with presence of air bronchograms. Subpleural curvilinear bands or bands of ground-glass opacities with or without consolidation in a tethered, arching pattern with small connections to the pleura are also considered typical. Thickening of vessels within areas of lung pathology is classic and frequently found in all other confirmatory patterns. CO-RADS 5 coincides with Typical Appearance of RSNA Consensus on CT reporting [24], which encompasses areas of ground-glass opacities in a bilateral peripheral distribution with presence or absence of consolidations or interstitial septal thickening (crazy paving appearance).

The category CO-RADS 6 indicates RT-PCR-proven cases of COVID-19.

CO-RADS has shown an excellent inter-observer agreement, with the highest for categories CO-RADS 1 and 5.

\section{Pros and Cons of Standardized Reporting}

Standardized reporting provides radiologists with the guidance for reporting and clarity and better communication with referring doctors. Standardized lexicon provides better opportunity for future education, research and data mining. On the flipside, imaging findings of COVID-19 are relatively non- specific and may overlap with many other pathologies, including viral pneumonias like Influenza-A. Positive predictive value of standardized reporting may be higher in populations with established community transmission. It is also noteworthy that many COVID-19 patients may have superimposed findings of secondary pathologies which may complicate the diagnosis.

\section{Conclusion}

As COVID-19 spreads across multiple nations, with many being limited in resources with respect to availability of tests and medical resources and with the resultant need to triage and effectively manage the available resources, imaging acquires an essential role. Many guidelines have emerged, which can guide us on indications of imaging in various clinical settings and triage of elective and emergency cases so as to manage radiology case-load. Protective precautions while imaging suspected or confirmed COVID-19 cases are especially important for protection of healthcare workers. CT imaging may reveal suspicious findings in asymptomatic patients. CORADS grading and RSNA Consensus on CT reporting can guide the disease risk based on imaging findings and be beneficial for effectively communicating the same to the physician. Radiologists must be aware of all the published guidelines to ensure optimal management of these cases while minimizing risk to healthcare workers.

Contributions The manuscript was written entirely by the authors, and all authors made an equal contribution in the development of the paper.

\section{Compliance with Ethical Standards}

Conflict of Interest The authors declare that they have no conflict of interest.

Ethics Declarations There are no sensitive data, and no patients were recruited for this study. The document does not conflict with ethical legislation.

\section{References}

1. Huang C, Wang Y, Li X, Ren L, Zhao J, Hu Y, et al. Clinical features of patients infected with 2019 novel coronavirus in Wuhan, China [published correction appears in Lancet. 2020 Jan 30;:]. Lancet. 2020;395(10223):497-506. https://doi.org/10. 1016/S0140-6736(20)30183-5.

2. Hui DS, Azhar EI, Madani T, et al. The continuing 2019-nCoV epidemic threat of novel coronaviruses to global health-the latest 2019 novel coronavirus outbreak in Wuhan, China. Int J Infect Dis. 2020;91:264-46.

3. World Health Organization (2020) WHO Director-General's remarks at the media briefing on 2019-nCoV on 11 February 2020. World Health Organization. Available via https://www.who.int/dg/ 
speeches/detail/who-director-generals-remarks-at-themediabriefing-on-2019-ncov-on-11-february-2020. Accessed 04 Aug 2020.

4. Wang W, Xu Y, Gao R, Lu R, Han K, Wu G, et al. Detection of SARS-CoV-2 in different types of clinical specimens [published online ahead of print, 2020 Mar 11]. JAMA. 2020;323(18):18434. https://doi.org/10.1001/jama.2020.3786.

5. Zou L, Ruan F, Huang M, Liang L, Huang H, Hong Z, et al. SARSCoV-2 viral load in upper respiratory specimens of infected patients. N Engl J Med. 2020;382(12):1177-9. https://doi.org/10. 1056/NEJMc20017376.

6. Fang Y, Zhang H, Xie J, Lin M, Ying L, Pang P, et al. Sensitivity of chest CT for COVID-19: comparison to RT-PCR. Radiology. 2020;296(2):E115-7. https://doi.org/10.1148/radiol.2020200432.

7. Radiological Society of North America. RSNA COVID-19 Task Force: best practices for radiology departments during COVID-19. 2020. https://www.rsna.org//media/Files/RSNA/Covid-19/RSNACOVID-19-bestpractices . as hx ? 1 a =en \& has h $=$ 58700DDDEDB3E5A9C8EDE80BE534B4ABB10291B7. Accessed 5 June 2020.

8. Society of Interventional Radiology. Aerosol generating procedures performed by interventional radiology clinical notification from the Society of Interventional Radiology. 2020. https:/www.sirweb. org/practice-resources/covid-19-resources/covid-19-clinicalnotifica-tion-3-26-20/. Accessed 15 June 2020.

9. Centers for Disease Control and Prevention. CDC statement for healthcare personnel on hand hygiene during the response to the international emergence of COVID-19. 2020. https://www.cdc. gov/coronavirus/2019-ncov/hcp/hand-hygiene.html?CDC_AA refVal=https $\% 3 \mathrm{~A} \% 2 \mathrm{~F} \% 2 \mathrm{Fwww} . \mathrm{cdc}$.gov $\% 2 \mathrm{~F}$ coronavirus $\%$ 2F2019-ncov\%2Finfection-control\%2Fhcp-hand-sanitizer.html. Accessed 10 June 2020.

10. Centers for Disease Control and Prevention. Airborne contaminant removal. 2019. https://www.cdc.gov/infectioncontrol/guidelines/ environmental/appendix/air.html\#tableb. Accessed 7 May 2020.

11. Laghi A, Grassi R. Italian Radiology's response to the COVID-19 outbreak. J Am Coll Radiol. 2020;17(6):699-700. https://doi.org/ 10.1016/j.jacr.2020.04.012.

12. Rubin GD, Ryerson CJ, Haramati LB, Sverzellati N, Kanne JP, Raoof S, et al. The role of chest imaging in patient management during the COVID-19 pandemic: a multinational consensus statement from the Fleischner society. Chest. 2020;158(1):106-16. https://doi.org/10.1016/j.chest.2020.04.003.

13. World Health Organization. Critical preparedness, readiness and response actions for COVID-19. https://www.who.int/ emergencies/diseases/novel-coronavirus-2019/technical-guidance/ critical-preparedness-readiness-and-response-actionsfor-covid-19. Updated March 22, 2020. Accessed Aug 4, 2020.

14. Wu Z, McGoogan JM. Characteristics of and important lessons from the coronavirus disease 2019 (COVID-19) outbreak in
China: summary of a report of 72314 cases from the Chinese Center for Disease Control and Prevention [published online ahead of print, $2020 \mathrm{Feb} 24]$. JAMA. 2020. https://doi.org/10.1001/jama. 2020.2648 .

15. Oba Y, Zaza T. Abandoning daily routine chest radiography in the intensive care unit: meta-analysis. Radiology. 2010;255(2):386-95. https://doi.org/10.1148/radiol.10090946.

16. Hejblum G, Chalumeau-Lemoine L, Ioos V, Boëlle PY, Salomon $\mathrm{L}$, Simon T, et al. Comparison of routine and on-demand prescription of chest radiographs in mechanically ventilated adults: a multicentre, cluster-randomised, two-period crossover study. Lancet. 2009;374(9702):1687-93. https://doi.org/10.1016/S01406736(09)61459-8.

17. Lakhal K, Serveaux-Delous M, Lefrant JY, Capdevila X, Jaber S, AzuRéa network for the RadioDay study group. Chest radiographs in 104 French ICUs: current prescription strategies and clinical value (the RadioDay study). Intensive Care Med. 2012;38(11): 1787-99. https://doi.org/10.1007/s00134-012-2650-9.

18. Suh RD, Genshaft SJ, Kirsch J, Kanne JP, Chung JH, Donnelly EF, et al. ACR Appropriateness Criteria ${ }^{\circledR}$ intensive care unit patients. J Thorac Imaging. 2015;30(6):W63-5. https://doi.org/10.1097/RTI. 0000000000000174.

19. Mizumoto K, Kagaya K, Zarebski A, Chowell G. Estimating the asymptomatic proportion of coronavirus disease 2019 (COVID-19) cases on board the Diamond Princess cruise ship, Yokohama, Japan, 2020. Euro Surveill. 2020;25(10):2000180. https://doi.org/ 10.2807/1560-7917.ES.2020.25.10.2000180.

20. Nishiura H, Kobayashi T, Miyama T, Suzuki A, Jung SM, Hayashi $\mathrm{K}$, et al. Estimation of the asymptomatic ratio of novel coronavirus infections (COVID-19). Int J Infect Dis. 2020;94:154-5. https://doi. org/10.1016/j.ijid.2020.03.020.

21. Caruso D, Zerunian M, Polici M, Pucciarelli F, Polidori T, Rucci C, et al. Chest CT features of COVID-19 in Rome, Italy. Radiology. 2020;296(2):E79-85. https://doi.org/10.1148/radiol.2020201237.

22. Caruso D, Polidori T, Guido G, et al. Typical and atypical COVID19 computed tomography findings. World J Clin Cases. 2020;8(15):3177-87. https://doi.org/10.12998/wjcc.v8.i15.3177.

23. Prokop M, van Everdingen W, van Rees VT, et al. CO-RADS: a categorical CT assessment scheme for patients suspected of having COVID-19-definition and evaluation. Radiology. 2020;296(2): E97-E104. https://doi.org/10.1148/radiol.2020201473.

24. Simpson S, Kay FU, Abbara S, et al. Radiological Society of North America Expert Consensus statement on reporting chest CT findings related to COVID-19. Endorsed by the Society of Thoracic Radiology, the American College of Radiology, and RSNA. Radiol Cardiothorac Imaging. 2020;2(2):e200152.

Publisher's Note Springer Nature remains neutral with regard to jurisdictional claims in published maps and institutional affiliations. 